\title{
Research on Responsibility Recognition and Calculation Method of Harmonic Active Power
}

\author{
Bin Yang \\ North China Electric Power University, Baoding 071000, China \\ 544635057@qq.com
}

Keywords: Power quality, Dominant harmonic source, Power quality monitoring system.

Abstract. The key of harmonic pollution control in power grid lies in the identification of harmonic pollution responsibility. According to the traditional harmonic voltage responsibility index to implement economic accounting to the defects, puts forward a definition to the true value of the harmonic active power, as the responsibility of the harmonic measure, and the calculation methods were also discussed.In the discussion process according to the equivalent circuit principle and the superposition theorem theory, and with the relative phase angle difference double leading method based on wave impedance, finally sorted out the complete definition and calculation method of harmonic active power responsibility. Through the measured case calculation, harmonic active power responsibility index can not only rational attention responsibility side harmonic pollution of harmonic sources, but also has the advantages of simple calculation, is conducive to economic accounting.

\section{Traditional harmonic responsibility index}

The first building concerns general bus model in figure 1. Assumes that the user $\mathrm{A}$ is nonlinear load are concerned, the definition of user side contains only focus on load A, the rest as the system side. Using harmonic voltage responsibility index $\mathrm{A}$ in the user finds the common connection point harmonic responsibility allocation.

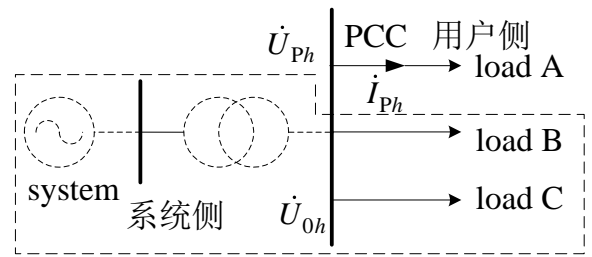

Fig. 1 General bus model of the concerned point

According to the superposition theorem, the distortion produced by harmonic voltage at PCC can be regarded as the result of the common contribution between the user side harmonic and the background harmonic source:

$\dot{U}_{P h}=\dot{U}_{A h}+\dot{U}_{0 h}$

The phasor quantities A, B and C satisfy the relation shown in Fig. 2. According to the definition of the harmonic voltage responsibility, the harmonic voltage responsibility index A of the load mAh can be calculated, as shown in equation (2):

$$
\mu_{A h}=\frac{U_{A h} \cos \theta}{U_{P h}} \times 100 \%
$$

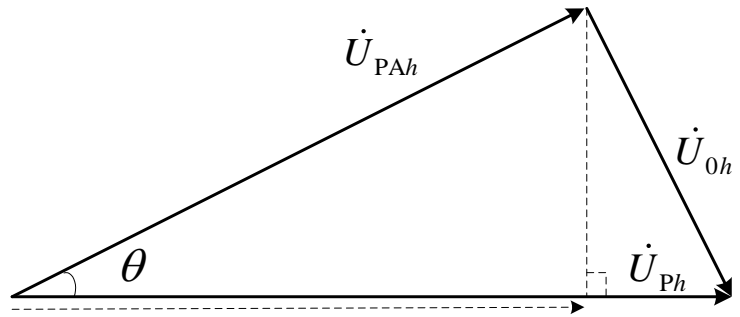

Fig. 2 Relationship of harmonic voltage

According to the traditional harmonic voltage responsibility index, can judge the harmonic harmonic distortion of public concern at the connection point can increase or inhibition, and the 
quantitative determination of attention on the side of the public responsibility of harmonic pollution at the connection point. But obviously, based on the index of pollution liability liability obtained by the harmonic voltage can not implement economic accounting, allocation therefore, the index is still unable to effectively solve the harmonic pollution liability.

\section{Method for calculating harmonic active power responsibility}

When the user side harmonic dominates, the calculation method of harmonic active power responsibility is summarized as the following three steps:

In the first step, the equivalent harmonic model of background harmonic source is established to calculate the equivalent impedance of the system side. The second step is to solve the equivalent harmonic current injected by the background harmonic source at the common connection point. The third step is to calculate the harmonic active power responsibility of the user side

\section{PSCAD simulation}

In order to verify the correctness of the above calculation method, this section uses PSCAD simulation to verify the true value of a harmonic current injected at a PCC of a simple system equivalent circuit.

In practical engineering, through calculation and analysis of power system, the equivalent to PCC node for the lumped parameter circuit, the system side and user side were reduced to the Thevenin and Norton equivalent circuit. The simulation experiment is to verify the accuracy of the constant calculation method, the key is to verify the correctness of the harmonic current calculation of the injected value, so the analog circuit should have the following two conditions:

1.The parameters in an equivalent system are easily measured or calculated;

2.True current injection values at PCC can be accurately compared

Here, a general lumped parameter distribution model of the power supply system is constructed, as shown in Figure 3:

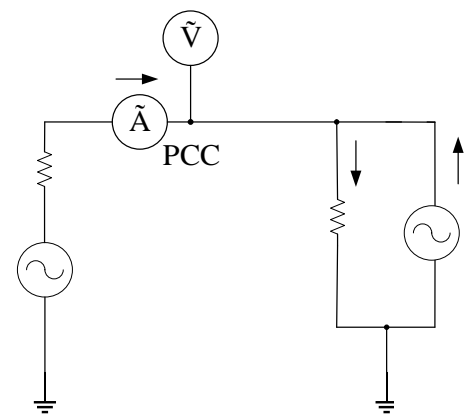

Fig. 3 The h'th harmonic circuit simulation diagram of power system

Note that the circuit model for a single $(\mathrm{H})$ equivalent circuit, the power system side, the background harmonic source voltage are $\mathrm{h}$ harmonic components, the equivalent impedance is the impedance value of the $\mathrm{H}$ harmonics. After the simulation of the query data set parameters in the circuit and the set value is shown in Table 1.

Table 1. Parameter setting value and measured value in simulation system

\begin{tabular}{ccc}
\hline System index & Effective value & Vector relative phase angle \\
\hline$\dot{U}_{o h}$ & $300 \mathrm{~V}$ & $83^{\circ}$ \\
$\dot{U}_{p c c}$ & $120.84 \mathrm{~V}$ & $-2226.1^{\circ}$ \\
$\dot{I}_{p c c}$ & $9.92 \mathrm{~A}$ & $89315.06^{\circ}$ \\
$\mathrm{Z}_{h}$ & $42.19 \Omega$ & $5.4677^{\circ}$ \\
$\mathrm{Z}_{A h}$ & $223.6 \Omega$ & $26.5651^{\circ}$ \\
$\dot{I}_{s h}$ & $0.1221 \mathrm{~A}$ & $84751.86^{\circ}$ \\
$\dot{I}_{\text {real }}$ & $9.80 \mathrm{~A}$ & $89371.85^{\circ}$ \\
\hline
\end{tabular}

Based on the above parameters, the actual harmonic current injection value at PCC is calculated by the calculation method mentioned above, and the error is compared with the measured value. 
Table 2 Comparison of calculated and measured result

\begin{tabular}{cccc}
\hline Research quantity & Calculated value & Measured value & Degree of deviation \\
\hline$\dot{I}_{\text {sh }}$ & $0.1139 \mathrm{~A}$ & $0.1221 \mathrm{~A}$ & $6.720 \%$ \\
$\dot{I}_{\text {real }}$ & $9.67 \mathrm{~A}$ & $9.80 \mathrm{~A}$ & $1.327 \%$ \\
\hline
\end{tabular}

From Table 2 the calculation results can be considered at the user side current harmonic PCC is proposed in this paper. The calculation method of $\dot{\boldsymbol{I}}_{\text {real }}$ value into the real right, the theory can be obtained accurately into the real value of the user side of the system side harmonic current. On this basis, to further ensure that the calculated user side dominant situation of rationality, harmonic active power under the responsibility of the dominant harmonic source at the PCC point of the fair.

\section{Conclusion}

According to the harmonic pollution liability in power system, this paper proposes a harmonic active power responsibility index and its definition and calculation method are discussed. Through theoretical analysis and case calculation, summarized as follows:

1) Compared with the traditional harmonic voltage responsibility, the advantage of the harmonic active power responsibility index is that the index can be implemented in the economic accounting, which is conducive to the implementation of harmonic pollution incentives and penalties, and is of great significance to the power quality management

2) From the definition and calculation method characteristics analysis, harmonic active power index should pay attention to three points in practical application:

First, the responsibility index is based on the true value of harmonic active power measurement as a reference value. Considering the harmonic alternating effect between the system side and user side, in the calculation of harmonic active power responsibility through an iterative algorithm to calculate the approximate value of the metal part, harmonic active power so as to obtain the user's real value.

Second, calculate the responsibility index needs to lead the harmonic source tracking accuracy and accurate estimation of harmonic impedance based on the equivalent condition. Calculation method of responsibility index in different conditions need to be adjusted accordingly, but the theory does not make roughly the same, here discussed. The harmonic impedance estimation accuracy is related to the accuracy and fairness index to determine the pollution liability.

Third, when the composition of harmonic components is more complex, it is necessary to calculate the active power of each harmonic separately, and then stack to obtain the responsibility of the active power of the harmonic at the attention side.

There is no doubt that the harmonic responsibility this index has a positive effect on the existing incentive programs, incentive programs can be rationally designed according to the [1] [8] index. It can be said that the harmonic active power index put forward in this paper is a simple and effective harmonic pollution liability index, power to an important role in promoting quality management of the will of the power system.

\section{References}

[1] Mceachern A, Grady W M, Moncrief W A, et al. Revenue and harmonics: an evolution of some proposed rate structures [J]. IEEE Transactions on Power Delivery, 1995, 10(1): 474-482.

[2] IEEE Std. 519-1992: Recommended Practices and Requirements for Harmonic Control in Electric Power Systems [S]

[3] IEC 61000-3-6, 1996: Assessment of emission limits for distorting loads in MV and HV power systems, technical report type 3 [S]

[4] IEC 61000-3-2, 2001-10: Electromagnetic compatibility (EMC)-part 3-2: limits-limits for harmonic current emissions (equipment input current $<=16 \mathrm{~A}$ per phase) $[\mathrm{S}]$

[5] IEC 61000-2-12, 2003-04: Electromagnetic compatibility (EMC)-part 2-12: compatibility levels for low-frequency conducted disturbances and signaling in public medium-voltage power supply systems $[\mathrm{S}]$ 